\title{
A new anatomical insight into the aetiology of lateral trunk of suprascapular nerve neuropathy: isolated infraspinatus atrophy
}

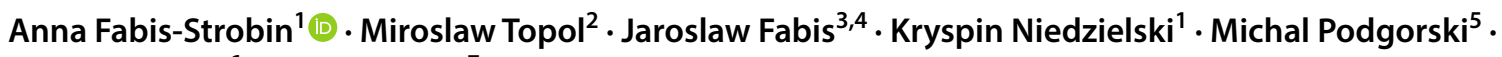 \\ Lukasz Strobin $^{6} \cdot$ Michal Polguj $^{7}$
}

Received: 8 October 2017 / Accepted: 26 February 2018 / Published online: 9 March 2018

(c) The Author(s) 2018. This article is an open access publication

\begin{abstract}
Introduction Although the pathomechanism of isolated infraspinatus atrophy (ISA) in throwing sports is known to be traction, it is unclear why only some players are affected. One likely explanation is that the infraspinatus pulling force exerted by its contracture generate the compressive resultant component force (Fn) compressing the lateral trunk of the suprascapular nerve (LTSN) against the edge of scapular spine. This paper makes two key assumptions (1) the course of LTSN in relation to the scapular spine, defined as the suprascapular-scapular spine angle (SSSA) is the key individual anatomical feature influencing the Fn magnitude, and thus potentially ISA development (2) SSSA is correlated with scapular notch type.

Materials and methods The bone landmarks of the LTSN course were identified in 18 formalin-fixed cadaveric shoulders, and the SSSA was measured in 101 dry scapulae. The correlation between the SSSA and suprascapular notch type was evaluated. The Fn value was simulated mathematically based on the values of the SSSA of 101 dry scapulae and the prevalence of ISA in chosen throwing sports, as given in the literature: i.e., beach volleyball - 34\% (group A1 - 34\%; group A2-remaining $66 \%$ of scapulae) and tennis $-52 \%$ (group B1 $-52 \%$; group B2-remaining $48 \%$ of scapulae).

Results The mean SSSA value was $44.57^{\circ}( \pm 7.9)$ and Fn $79 \mathrm{~N}( \pm 13.1)$. No statistically significant correlation was revealed between suprascapular notch type and SSSA. Groups A1 and B1 possessed significantly lower SSSA values $(p<0.000)$ and significantly higher Fn magnitude $(p<0.000)$ than groups A2 and B2 respectively. The average difference of Fn was $28.1 \%$ between group $\mathrm{A} 1$ and $\mathrm{A} 2$ and $31 \%$ between group B1 and B2.

Conclusions The SSSA has a wide range of values depending on the individual: the angle influencing the magnitude of the compressive resultant force Fn on the LTSN at the lateral edge of the scapular spine via contraction of the infraspinatus muscle. The prevalence of ISA in throwing sports may be correlated with the SSSA of the LTSN. However, further combined clinical, MRI or/and CT studies are needed to confirm this.
\end{abstract}

Keywords Suprascapular neuropathy $\cdot$ Isolated infraspinatus atrophy $\cdot$ Throwing sports $\cdot$ Suprascapular nerve anatomy

\section{Abbreviations}

ISA Isolated infraspinatus atrophy

LTSN Lateral trunk of the suprascapular nerve

Anna Fabis-Strobin

anna.m.fabis@gmail.com

1 Clinic of Orthopaedic and Traumatology, Polish Mother's Memorial Hospital Research Institute, Rzgowska 281/289, 93-338 Lodz, Poland

2 Department of Normal and Clinical Anatomy, Interfaculty Chair of Anatomy and Histology, Medical University of Łódź, Narutowicza 60, 90-136 Lodz, Poland

3 Department of Arthroscopy, Minimally Invasive Surgery and Sports Traumatology, Medical University of Lodz, Zeromskiego 113, 90-549 Lodz, Poland
SSSA Suprascapular-scapular spine angle

Fn Resultant compressive force

$F \quad$ Pulling force of insfraspinatus contracture

4 FMC Medical Center, Pilsudskiego 9a, 90-368 Lodz, Poland

5 Department of Diagnostic Imaging, Polish Mother's Memorial Hospital Research Institute, Rzgowska 281/289, 93-338 Lodz, Poland

6 Faculty of Technical Physics, Information Technology and Applied Mathematics, Lodz University of Technology, Wolczanska 215, 90-924 Lodz, Poland

7 Department of Angiology, Interfaculty Chair of Anatomy and Histology, Medical University of Łódź, Narutowicza 60, 90-136 Lodz, Poland 


\section{Introduction}

Despite the passage of time, the aetiology of isolated atrophy of the infraspinatus (ISA) in throwing sports remains poorly understood. Its frequency of occurrence can be surprisingly high, reaching $34 \%$ in beach volleyball [22], with an average prevalence of 52\% among elite female tennis players [50]. It has been suggested that in these cases, its development occurs as a result of nerve traction during repeated overhead motion of the shoulder [4, 12, $17,21-23,28,34,36,39,42,46,47,50]$, however, it is unclear why only some players are affected. Therefore it seems the pathomechanism must be more complex and associated with the key anatomical player, i.e., the lateral trunk of the suprascapular nerve (LTSN) innervating the infraspinatus [16]. From a biomechanical point of view, the angle between the LTSN and the scapular spine, i.e., the suprascapular-scapular spine angle (SSSA), appears to have a key influence on the magnitude of the resultant compressive force (Fn) acting on the LTSN at the edge of the scapular spine [39] by pulling force $(F)$ exerted by contraction of the infraspinatus.

The aim of the present study was to clarify the biomechanical role of LTSN pulling in the development of ISA in throwing sports. To this end, the following secondary aims were pursued: (1) to identify the bone landmarks of the LTSN course in preparations of formalin-fixed cadaveric shoulders, (2) to measure the SSSA in dry scapulae, (3) to evaluate the occurrence of a particular type of scapular notch among the dry scapulae. (4) to calculate the Fn value based on the magnitude of SSSA with regard to the prevalence of ISA in chosen throwing sports, as given in the literature: i.e., beach volleyball $-34 \%$ [22] and tennis $-52 \%$ [50].

Based on the acquired data, the study verifies the following hypotheses: (1) the course of the LTSN in relation to the scapular spine, defined as the SSSA, is an individual anatomical feature and thus influences the magnitude of the compressive resultant force Fn of the infraspinatus pulling force $F$ exerted on it by infraspinatus muscle contraction; (2) the SSSA value depends on the type of scapular notch; (3) the acquired test results represent a valuable step forward to understanding ISA aetiology and act as a basis for further clinical research.

\section{Materials and methods}

The tests were performed in two stages: The first using 18 formalin-fixed, cadaveric shoulders and the second using 101 dry scapulae. All tested preparations, obtained from the collection of the Department of Normal and Clinical Anatomy of Medical University of Lodz, were undamaged, and taken from cadavers of unknown sex and age. The research project was approved by the Bioethics Commission of the Polish Mother's Memorial Hospital Research Institute (protocol no. 11/2017).

The upper limbs were examined to establish the bone landmarks of the LTSN course. In all preparations, a horizontal cut was made along the clavicle, and the skin was dissected from the deltoid, trapezius and pectoralis major. The deltoid and the trapezius were then removed. The upper edge of the scapula and the area around its notch were revealed by dissecting the supraspinatus muscle (Fig. 1). Next, based on the test results from the soft preparations of the upper limbs, the course of the LTSN was determined on the dry scapulae and the SSSA was measured. The SSSA is the angle included between the line running through the lowest point at the base of the scapular notch and the distal point of the scapular spine edge and the line joining the most prominent
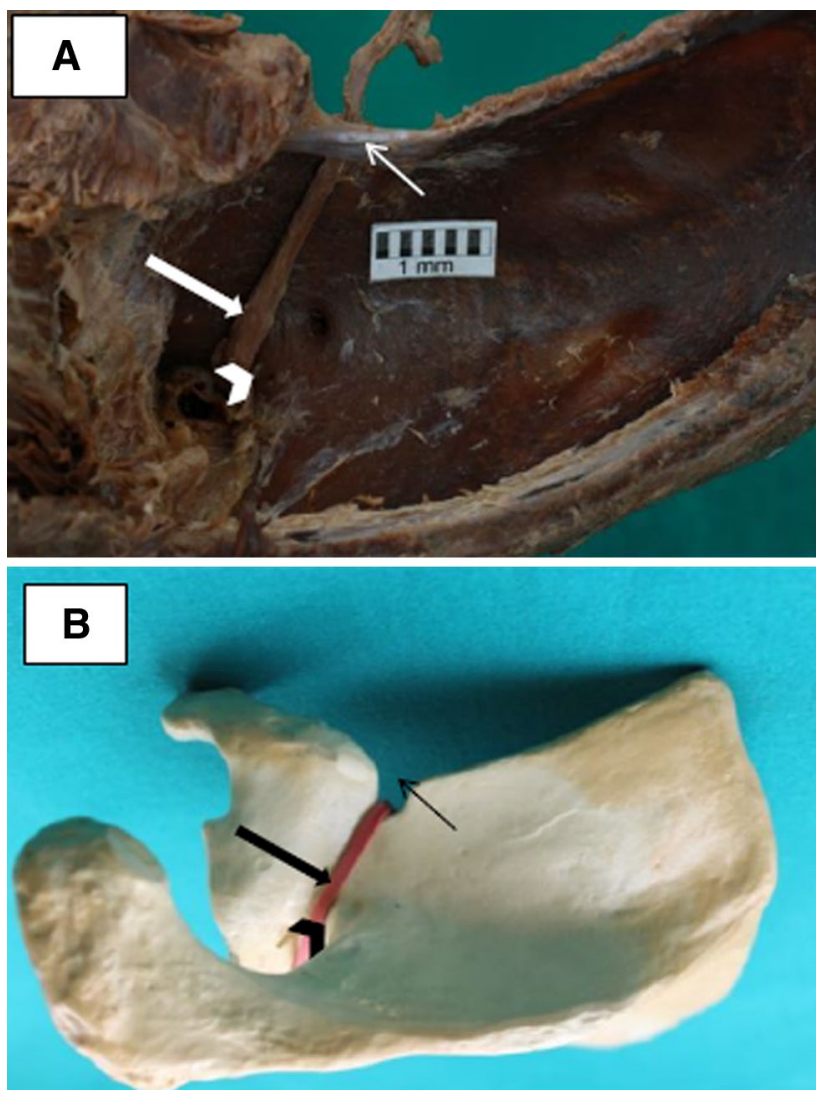

Fig. 1 a, b The course of the lateral trunk of the suprascapular nerve, confirmed by anatomical examination. a Permanent soft preparation of the upper limbs, posterior-superior view of the suprascapular fossa. b Dry preparation of the scapula indicating the course of the lateral trunk of the suprascapular nerve. Large arrow-lateral trunk of the suprascapular nerve, small arrow-superior transverse scapular ligament, arrowhead-lateral edge of the scapular spine 
medial and lateral points of superior aspect of scapular spine. The scapular spine arm of the tool used for SSSA measurement automatically positioned itself on the two most superior points (lateral and medial) of the scapular spine. The arm was set in fixed plastic material, allowing a precise reading to be taken of the angle between the arms of the tool (Fig. 2). The arrangement of the measuring tool arms indicated the course of the LTSN in the fixed preparation.

The SSSA was measured four times in an independent series of measurements performed every 2 weeks. The measurements were performed by the first author AFS ( 2 measurements) and co-author MP (2 measurements). The inter- and intra-observer reliability tests were done (interclass correlation coefficient). The mean value of the four measurements was used for the statistical analysis.

In the second stage, the 101 dry scapular preparations were classified according to notch-type classification based on measurements of its dimensions according to Polguj et al. [31] (Fig. 3; Table 1). All measurements were performed with an analogue calliper with a resolution of $0.05 \mathrm{~mm}$ (MITUTOYO, Japan).

To examine the hypothesis that the SSSA value influences the risk of lateral trunk suprascapular neuropathy (LTSN) four groups were separated from the 101 scapulae under investigation-A1, A2, B1 and B2. Group A1 and B1 were formed to include the proportion of scapulae from the total test sample which reflects the proportion of scapulae demonstrating LTSNN in beach volleyball players (34\% of 101 scapulae; $n=34$ ) [22], and in tennis players (52\% of 101 scapulae; $n=52$ ) [50]. In addition, two groups were formed to reflect the proportions of players who do not suffer from LTSNN: Group A2 for the beach volleyball players $(n=67$ scapulae [101-34]) and Group B2 for the tennis players ( $n=49$ scapulae [101-52]). The statistical

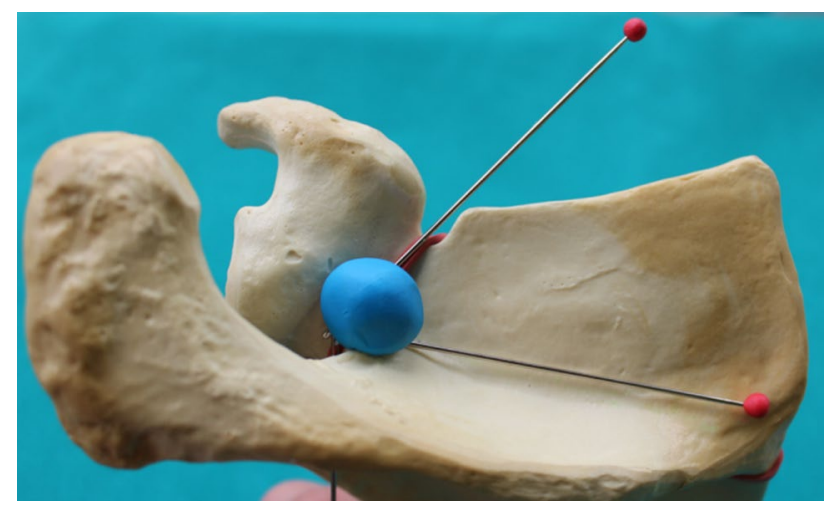

Fig. 2 The tool used for measuring the suprascapular spine angle (SSSA), and the means by which the angle was determined with regard to the reference points on the bone (bottom of scapular notch, lateral point of scapular spine edge, medial and lateral points of upper aspect of scapular spine), and the method of preserving the position of the measuring device using plastic materials

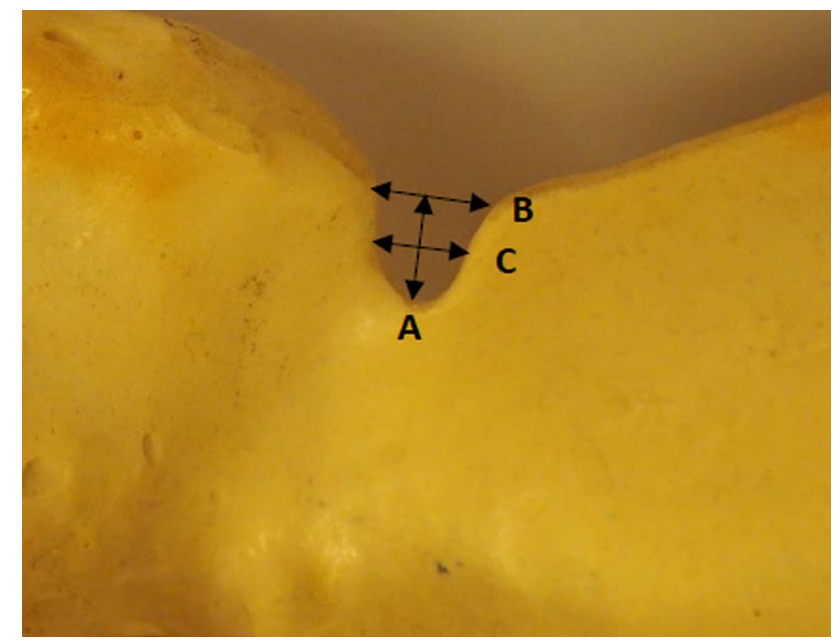

Fig. 3 Suprascapular notch dimension measurements of maximal depth a; superior transverse $\mathbf{b}$; middle transverse $\mathbf{c}$ according to classification proposed by Polguj et al. [31]

analysis examined the comparison between groups $\mathrm{A} 1$ and $\mathrm{A} 2$ and groups B1 and B2.

The compressive force acting on the LTSN (Fn) against the scapular spine edge is the resultant compressive component of the pulling force exerted by the contraction of the infraspinatus $(F)$ (Fig. 4) [13]. To calculate the hypothetical magnitude of Fn of pulling force $F$ of infraspinatus, the appropriate physical formula was used [13]. The magnitude of the infraspinatus pulling force $(F)$, i.e. $205 \mathrm{~N}$, was taken from a study by Escamila et al. [8]. Therefore, the hypothetical calculation of force Fn was based on the following three components: (1) The physical formula $\left[\mathrm{Fn}=F \sin \frac{\left(90^{\circ}-\mathrm{SSSA}\right)}{2}\right]$, derived by taking the force component perpendicular to the edge [13]; (2) the average value of infraspinatus force $(F)$ according to Escamila et al. [8] i.e., $205 \mathrm{~N}$; (3) the value of SSSA evaluated in the present study.

Table 1 Suprascapular notch classification based on the notch dimension measurements of maximal depth (A); superior transverse (B); middle transverse (C) according to Polguj et al. [31]

\begin{tabular}{ll}
\hline Notch type & Classification criteria \\
\hline I & General $A>B$ \\
& Ia $B<C ;$ Ib $B=C ;$ Ic $B>C$ \\
II & $A=B=C$ \\
III & General $A<B$ \\
& IIIa $B<C ;$ IIIb $B=C ;$ IIIc $B>C$ \\
IV & Bony foramen \\
V & Discrete notch \\
\hline
\end{tabular}




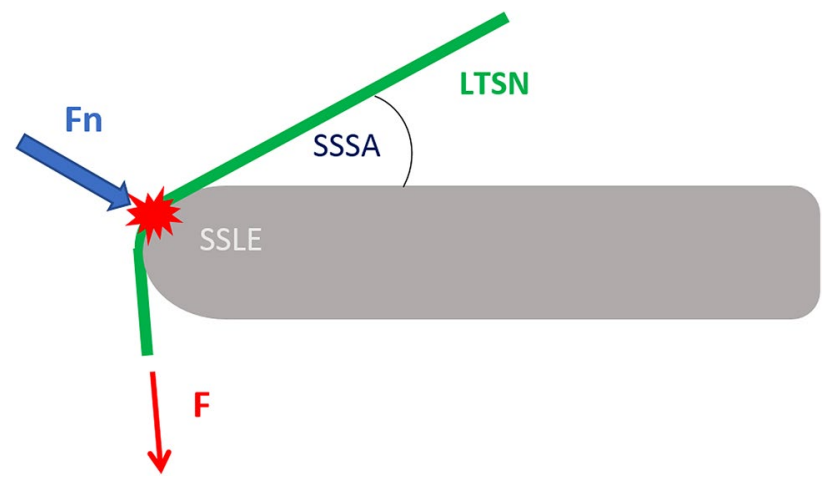

Fig. 4 The schematic drawing of resultant compressive force (Fn) of the pulling force of infraspinatus contraction $(F)$, acting on the lateral trunk of the suprascapular nerve (LTSN) against the scapular spine lateral edge (SSLE). SSSA suprascapular spine angle

\section{Statistical analysis}

A power analysis was performed ( $\alpha=0.05,80 \%$ power). The effect size $(d)$ of the differences were calculated (small if $0 \leq|d| \leq 0.5$, medium if $0.5,|d| \leq 0.8$, and large if $|d|>0.8$ ). The intra- and inter-observer correlation (interclass correlation coefficient) were calculated for the four measurements of SSSA. The normality of the data was evaluated using the Shapiro-Wilk test. Student's $t$ test was used for normally distributed data with equal variances, and the $U$ Mann-Whitney test for non-normally distributed data. $p$ values below 0.05 were regarded as statistically significant.

All calculations were performed with STATISTICA ver10. (StatSoft, Inc, Pl, 2011; http://www.statsoft.com) The correlation between the SSSA and suprascapular notch type was calculated using Weka 3: Data Mining Software in Java [15] designed for data mining and detection of complex relationships between the analyzed values.

Table 2 The SSSA values according to four independent measurements performed by first author (measurements No. 1 and No. 2) and co-author (measurements No. 3 and No. 4)

\begin{tabular}{lllll}
\hline $\begin{array}{l}\text { Number of } \\
\text { measure- } \\
\text { ment }\end{array}$ & Mean value & $\begin{array}{l}\text { Minimum } \\
\text { value }\end{array}$ & $\begin{array}{l}\text { Maximum } \\
\text { value }\end{array}$ & $\begin{array}{l}\text { Standard } \\
\text { deviation }\end{array}$ \\
\hline No. 1 & 43.08 & 25.00 & 67.00 & 8.65 \\
No. 2 & 45.53 & 26.00 & 65.00 & 7.98 \\
No. 3 & 44.66 & 25.00 & 74.00 & 9.04 \\
No. 4 & 45.02 & 25.00 & 68.00 & 7.87 \\
\hline
\end{tabular}

Table 3 The extended comparison (interclass correlation coefficient-ICC) between 4 measurements of SSSA performed independently by first author (measurements No. 1 and No. 2) and co-author (measurements No. 3 and No. 4)

\section{Results}

The sample size of 101 scapulae provided $>90 \%$ power for all comparisons. The effect size of the differences was large for all comparisons $(d>0.8)$. Table 2 presents data of four independent measurements of SSSA, and Table 3 contains the extended statistical analysis of comparison between these measurements (interclass correlation coefficient). The mean SSSA value was $44.57^{\circ}\left(28.5^{\circ}-67.25^{\circ} \pm 7.9\right)$. The most common type of suprascapular notch was Type III (Table 4). No statistically significant correlation was found between notch type and SSSA value (Weka 3 analysis) (Table 4). However, it was found that the SSSA value of group A1 was significantly lower than in group A2 ( $U$ Mann-Whitney test, $p<0.000$ ) and an analogous difference was found between groups B 1 and $\mathrm{b}(p<0.000, U$ Mann-Whitney test) (Table 5). The mean calculated force exerted by the infraspinatus $(\mathrm{Fn})$ on the LTSN was $79 \mathrm{~N}(40.43 \mathrm{~N}-104.81 \mathrm{~N} \pm 13.1)$ with a nearly linear graph (Fig. 5). Moreover, our mathematical simulation revealed significant differences of Fn between groups $\mathrm{A} 1$ and $\mathrm{A} 2(p<0.000, U$ Mann-Whitney test $)$ and between groups $\mathrm{B} 1$ and $\mathrm{b}(p<0.000, U$ Mann-Whitney test $)$ (Table 5). The average difference of Fn was $28.1 \%$ between groups A1 and A2 and $31 \%$ between groups B1 and B2 (Table 5).

\section{Discussion}

The most important finding of our study is the disclosure of a potential relationship between the anatomical course of LTSN, expressed as SSSA and the aetiology of ISA in throwing sports. Neurogenic ISA affects between 4.4 and $52 \%$ of baseball, volleyball and female tennis players [4, $17,22,46,50]$.

This high frequency of occurrence and the fact that the condition is not found in all players are not accounted for by the proposed pathomechanisms of ISA, in which a crucial role is played by hyper-abduction $[4,12,17,21-23,28,34$, 
Table 4 Values of SSSA angle $\left({ }^{\circ}\right)$ against notch type according to Polguj et al. [31]

\begin{tabular}{lllllcc}
\hline Notch type & Mean value & Minimum value & Maximum value & $\begin{array}{l}\text { Standard } \\
\text { deviation }\end{array}$ & $\begin{array}{l}\text { Number } \\
\text { of scapu- } \\
\text { lae }\end{array}$ & \% of scapulae (\%) \\
\hline Type I & 45.82 & 32.75 & 62.0 & 8.12 & 14 & 13.86 \\
Type II & 43.72 & 37.0 & 50.25 & 5.4 & 8 & 7.92 \\
Type III & 44.47 & 28.75 & 67.25 & 7.73 & 59 & 58.41 \\
Type IV & 52.49 & 41.0 & 61.75 & 7.75 & 6 & 5.94 \\
Type V & 40.75 & 28.5 & 51.5 & 7.78 & 14 & 13.86 \\
\hline
\end{tabular}

Table 5 Mean value, range and standard deviation of the SSSA $\left({ }^{\circ}\right)$ and Fn (Newtons) calculated based on the literature prevalence of ISA in beach volleyball players (group A1, A2) and tennis players (group B1, B2)

\begin{tabular}{|c|c|c|c|c|}
\hline Group & Mean & Minimum & Maximum & $\begin{array}{l}\text { Standard } \\
\text { deviation }\end{array}$ \\
\hline A1-SSSA & 36.4 & 28.5 & 40.0 & 3.72 \\
\hline A2-SSSA & 48.73 & 40.0 & 67.25 & 6.02 \\
\hline B1-SSSA & 38.36 & 28.5 & 45.0 & 4.15 \\
\hline B2-SSSA & 51.17 & 45.0 & 67.25 & 5.13 \\
\hline A1-Fn & 92.4 & 86.63 & 104.81 & 5.9 \\
\hline A2-Fn & 72.15 & 40.43 & 86.63 & 10.15 \\
\hline B1-Fn & 89.22 & 78.45 & 104.81 & 6.62 \\
\hline B2-Fn & 68.1 & 40.43 & 78.45 & 8.75 \\
\hline \multicolumn{5}{|c|}{$\begin{array}{l}\text { Group A1-34\% of scapula with lowest SSSA; Group A2-the } \\
\text { remaining } 66 \% \text { of scapula }\end{array}$} \\
\hline \multicolumn{5}{|c|}{$\begin{array}{l}\text { Group B1-52\% of scapula with the lowest SSSA value; Group } \\
\text { B2 - the remaining } 48 \% \text { of scapula }\end{array}$} \\
\hline
\end{tabular}

$36,39,42,46,47,50]$, occasionally combined with specific scapular kinematics and increase of shoulder range of motion [47], rare anatomical variants [5, 7, 34, 39, 48] and the presence of cyst in the spinoglenoid notch [20,45]. Therefore, assuming that a relationship exists between suprascapular nerve neuropathy and damage to the rotator cuff $[2,3,25$, $26,40]$, there is a pressing need for more thorough analyses of the biomechanical conditions of LTSN function. No significant correlation was found between SSSA and notch type which was classified according to Polguj et al. [31] (Table 4). Furthermore, the frequency of occurrence of particular notch types was found to be generally in line with those identified in related publications [31-33]. Therefore, we assumed that it was suitable for our hypothetical simulation, which revealed significant differences between group $\mathrm{A} 1$ and A2, as well as between group B1 and B2 with regard to SSSA and Fn values.

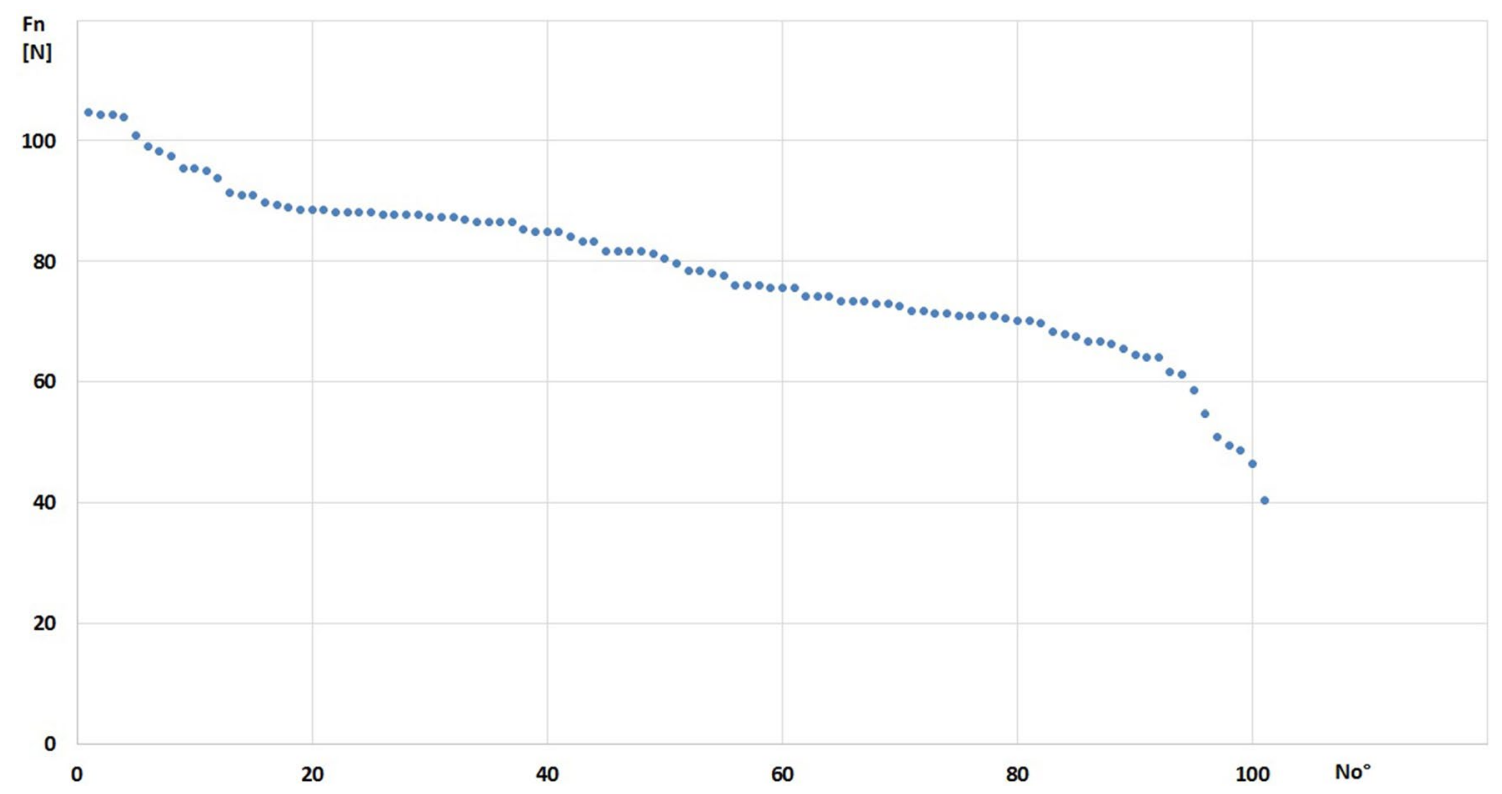

Fig. 5 The graph of the distribution of force Fn in the studied group of 101 scapulae 
Collectively, these findings indicate that the development of ISA in different throwing sports may depend on the SSSA value, but the frequency of occurrence is related to existing specific global biomechanical differences between the functional demands placed on the shoulder girdle. Volleyball players are known to complete at least 1000 serves per week [17], which may correspond to 40,000 overhead movements per year, as reported by Dubotzki et al. [6]. Furthermore, according to our mathematical simulation, the hypothetical cumulative mean Fn acting on the LTSN during the year (i.e., the number of serves and spikes $\times 79 \mathrm{~N}$ ) would be $3,160,000 \mathrm{~N}$. These figures are clearly unbelievable, and in the case of Group A1, even more so (Table 5). From a pathophysiological perspective, ISA is one of the group of chronic nerve compression injuries of the upper extremities which arises in response to intermittent chronic compression, leading to disturbances in the blood supply to the LTSN $[35,43]$. During the early stage of ISA development, compression of the LTSN vessels leads to temporary epineural ischemia [35] which, in the case of volleyball, may be manifested as pathological EMG findings [22]. The chronicity of ISA leads to fibrosis, demyelination and LTSN fibre degeneration $[17,22,35]$. This approach has a strong scientific base, since pathophysiology of chronic nerve compression injuries is independent of axonal damage [35]. Taking these findings together with ours, we propose a new term for isolated infraspinatus atrophy: in our view, the term neuropathy of the lateral trunk of the suprascapular nerve (LTSNN) is more appropriate as it defines the source of the pathology rather than simply describes the clinical symptom.

In case of throwing sports, Fn acts both as a concentric accelerating force during the windup and cocking phase of shoulder hyper-abduction, and as a decelerating eccentric force immediately afterwards $[37,38]$. Therefore, Fn prolongs intermittent disturbances of blood circulation within the LTSN vessels during the specific phases of throwing in spite of the presence of some differences in infraspinatus EMG activity characteristic of a particular throwing discipline $[37,38]$. It seems that the Fn exerted by the infraspinatus on LTSN during the decelerating stage is more dangerous than that exerted during acceleration, since it is associated with an increase in the passive stretch of the LTSN due to separation of the neck and scapula, however, this issue requires further study. Moreover, it should be stressed that some other co-existing mechanisms are possible: i.e., excessive range of motion of the shoulder girdle [47], specific scapular kinematics $[18,47]$ and their relationship to proprioception disturbance [10]. Our global biomechanical concept of LTSNN (ISA) development is summarised in Fig. 6.

The early diagnosis and treatment of LTSNN (ISA) is especially important because only partial recovery of isokinetic peak torque is possible in the external rotators following arthroscopic decompression of the suprascapular nerve

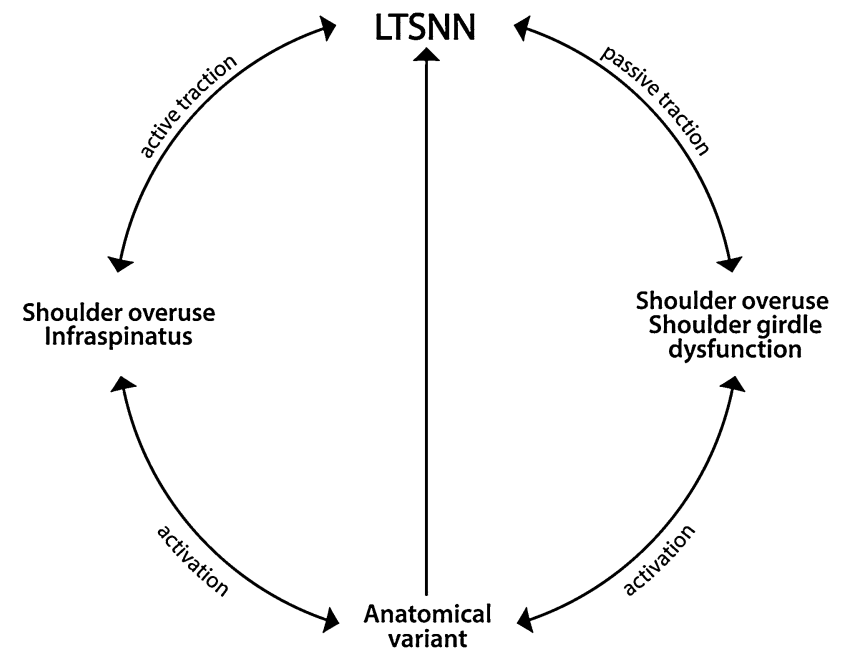

Fig. 6 The global biomechanical concept of the development of lateral trunk of suprascapular nerve neuropathy (LTSNN)

[11], as is the case with external rotator strength after open spinoglenoid notch plasty [39] and its decompression [24]. In the light of this pathophysiology [35], 5 the incomplete recovery of muscle atrophy [12] and external rotator strength $[24,39]$ indicates that the degeneration of some axons associated with an advanced stage of LTSNN development is irreversible. Furthermore, since the recovery of external rotator strength observed following proper training is also connected with hypertrophy of the teres minor $[19,49]$ the extent of irreversible degeneration of the LTSN axons may be more advanced than first thought.

As LTSNN does not induce any significant clinical shoulder dysfunction and the athlete may continue competing, the clinical significance of this condition is often overlooked $[12,17,22]$. Nevertheless, a wealth of epidemiological data exists concerning rotator cuff pathology and its prevalence is known to increase with age, from $9.7 \%$ in patients aged from $\leq 20$ years to $62 \%$ in those aged $\geq 80$ years [44].

Furthermore, atrophy and fatty infiltration of the supraspinatus is known to reach an irreversible stage at an average of 5 years after the onset of symptoms [27]. Taking these findings collectively, irreversible LTSN axon damage associated with rotator cuff tear is a poor prognostic clinical finding during a sporting career, and even more so after its completion [2]. Recent experimental $[9,30]$ and clinical data indicates that muscle atrophy is reversible following successful reconstruction of the rotator cuff; however, restoration of the infraspinatus will not be possible if accompanied by the irreversible stage of the LTSNN. The study has some limitations. The evaluation of the LTSN course may be subject to some error although it should be stressed that the method of SSSA measurement is based on stable bone landmarks determined at 18 formalin-fixed cadaveric shoulders and the 
extended evaluation of interclass correlation coefficient proved its reliability. In addition, the age, sex and range of differences side to side of the examined scapulae were not known. However, according to Paraskevas et al. [29] the shape of the acromion was symmetrical in $65.9 \%$ of cases, and their results reveal no correlation between shape and gender. Shi et al. [41] confirmed that there is excellent side-to-side symmetry in glenoid shape regardless of sex. Moreover, Polguj et al. [32] note that the distribution of the suprascapular notch types is similar between the two sexes. Sexual dimorphism was further investigated by Polguj et al. [33] on total of 616 computer tomography scans of shoulders randomly selected from the database of patients who were being investigated due to chest problems. This study revealed that the frequency of type I and IV was lower in females than in males, but type III was more common in females than males. The other types of suprascapular notch had similar distributions in the two sexes. Additionally, the authors revealed that from among three dimensions characterising the type of notch only the maximal notch depth was statistically significantly higher in males than in females [33]. In contrast to Polguj et al. [33], Albino et al. [1] reported no correlation between notch type and gender, as well as age. This study was done on 500 dry scapulae. However, it should be noted that the Albino et al. [1] study applied other than Polguj et al. [33] notch classification.

Furthermore, Gumina et al. [14] examined 500 dried scapulae, measuring six distances for each one (referring to the scapular body, glenoid, and the course of the suprascapular nerve). They found insignificant differences between the right and left scapulae, and significant differences between those taken from males and females. Therefore, based on all these findings, together with the lack of literature about the impact of correlation between the sex and particular parameters of the scapula on isolated infraspinatus atrophy prevalence in throwing sports it is seemed that age, sex and side have no important impact on the SSSA measurement and value. In addition, the contraction of the supraspinatus may also temporarily affect the course of the LTSN leading to the decrease of SSSA value [30], which was not taken into account. The mean value of the infraspinatus force $\mathrm{F}$ was related to its selective activity in common shoulder rehabilitation exercises [8]; despite this, the key issue is the SSSA value which finally influences the magnitude of Fn.

Nevertheless, despite these limitations, our findings represent an important contribution which deepens the understanding of the pathomechanism of LTSNN development in throwing sports. Moreover, it serves as a basis for determining MRI and CT protocols for SSSA evaluation, as well as for further clinical studies intended to highlight the method of selecting athletes at risk of LTSNN development, and its subsequent treatment in the case of occurrence.

1. The course of the lateral trunk of the suprascapular nerve, defined as the suprascapular-scapular angle, is a feature specific to an individual.

2. The results of the study revealed the potential relationship between the anatomical course of the lateral trunk of the suprascapular nerve expressed as the suprascapular-scapular angle and the aetiology of isolated infraspinatus atrophy development.

Acknowledgements Acknowledgements to prof. Henryk Małecki for consultations in the field of biomechanics.

Authors' contribution All authors confirm their contribution. FabisStrobin Anna data collection; data analysis; manuscript writing/editing; protocol/project development. Topol Miroslaw protocol/project development, manuscript revision. Fabis Jaroslaw data analysis; manuscript writing/editing; protocol/project development. Niedzielski Kryspin manuscript preparation. Podgorski Michal data collection. Strobin Lukasz data analysis; manuscript writing/editing, protocol/project development. Polguj Michal anatomical dissection, data analysis; manuscript revision; protocol/project development.

Funding None.

\section{Compliance with ethical standards}

Ethics approval and consent to participate The research conducted was in line with the consent of the bioethics committee, a consent to participate is not applicable.

Consent to publish Not applicable.

Availability of data and materials All data generated or analyzed during this study are included in this published article.

Conflict of interest The authors declare no conflict of interest.

Open Access This article is distributed under the terms of the Creative Commons Attribution 4.0 International License (http://creativeco mmons.org/licenses/by/4.0/), which permits unrestricted use, distribution, and reproduction in any medium, provided you give appropriate credit to the original author(s) and the source, provide a link to the Creative Commons license, and indicate if changes were made.

\section{References}

1. Albino P, Carbone S, Candela V, Arceri V, Vestri AR, Gumina S (2013) Morphometry of the suprascapular notch: correlation with scapular dimensions and clinical relevance. BMC Musculoskelet Disord 14:172. https://doi.org/10.1186/1471-2474-14-172

2. Beeler S, Ek ET, Gerber C (2013) A comparative analysis of fatty infiltration and muscle atrophy in patients with chronic rotator cuff tears and suprascapular neuropathy. J Shoulder Elbow Surg Vols 22(11):1537-1546. https://doi.org/10.1016/j.jse.2013.01.028 
3. Costouros JG, Porramatikul M, Lie DT, Warner JJ (2007) Reversal of suprascapular neuropathy following arthroscopic repair of massive supraspinatus and infraspinatus rotator cuff tears. Arthroscopy Vols 23(11):1152-1161. https://doi.org/10.1016/j. arthro.2007.06.014

4. Cummins CA, Messer TM, Schafer MF (2004) Infraspinatus muscle atrophy in professional baseball players. Am J Sports Med 32(1):116-120. https://doi.org/10.1177/0363546503260731

5. Demirhan M, Imhoff AB, Debski RE, Patel PR, Fu FH, Woo SL (1998) The spinoglenoid ligament and its relationship to the suprascapular nerve. J Shoulder Elbow Surg 7(3):238-243

6. Dubotzky V, Leistner M (1992) Volleyball. In: Ballreich R, Kuhlow-Balireich A Biomechanik der sportspiele: vol 2. Enke Verlag, Stuttgart, pp 72-123

7. Duparc F, Coquerel D, Ozeel J, Noyon M, Gerometta A, Michot C (2010)Anatomical basis of the suprascapular nerve entrapment, and clinical relevance of the supraspinatus fascia. Surg Radiol Anat 32(3):277-284. https://doi.org/10.1007/s00276-010-0631-7

8. Escamilla RF, Yamashiro K, Paulos L, Andrews JR (2009) Shoulder muscle activity and function in common shoulder rehabilitation exercises. Sports Med 39(8):663-685. https://doi. org/10.2165/00007256-200939080-00004

9. Fabis J, Danilewicz M, Zwierzchowski JT, Niedzielski K (2016) Atrophy of type I and II muscle fibers is reversible in the case of grade $>2$ fatty degeneration of the supraspinatus muscle: an experimental study in rabbits. J Shoulder Elbow Surg 25(3):487492. https://doi.org/10.1016/j.jse.2015.08.034

10. Fabis J, Rzepka R, Fabis A, Zwierzchowski J, Kubiak G, Stanula A, Polguj M, Maciej R (2016) Shoulder proprioception-lessons we learned from idiopathic frozen shoulder. BMC Musculoskelet Disord 17:123. https://doi.org/10.1186/s12891-016-0971-5

11. Fabis J (2007) Isokinetic evaluation of external rotators and abductors of the shoulder before and after arthroscopic decompression of suprascapular nerve due to its neuropathy-preliminary report. Chir Narzadow Ruchu Ortop Po 72:283-285

12. Ferretti A, De Carli A, Fontana M (1998) Injury of the suprascapular nerve at the spinoglenoid notch. The natural history of infraspinatus atrophy in volleyball players. Am J Sports Med 26(6):759-763

13. Feynman R, Leighton R, Sands M (1963) The Feynman lectures on physics, 6th printing. Addison-Wesley Publishing Company, Reading Masachusetts Califirnia, pp 91-100; 110-119

14. Gumina S, Albino P, Giaracuni M, Vestri A, Ripani M, Postacchini $F$ (2011) The safe zone for avoiding suprascapular nerve injury during shoulder arthroscopy: an anatomical study on 500 dry scapulae. J Shoulder Elbow Surg 20(8):1317-1322. https:// doi.org/10.1016/j.jse.2011.01.033

15. Hall M, Frank E, Holmes G, Pfahringer B, Reutemann P, Witten IH (2009) The WEKA data mining software: an update; SIGKDD Explorations 1/11. https://doi.org/10.1145/1656274.1656278

16. Hermenegildo JA, Roberts SL, Kim SY (2014) Innervation pattern of the suprascapular nerve within supraspinatus: a three-dimensional. Computer Modeling Study 27:622-630

17. Holzgraefe M, Kukowski B, Eggert S (1994) Prevalence of latent and manifest suprascapular neuropathy in high-performance volleyball players. Br J Sports Med 28(3):177-179. https://doi. org/10.1002/ca.22250

18. Kibler WB, Sciascia A (2010) Current concepts: scapular dyskinesis. Br J Sports Med 44(5):300-305. https://doi.org/10.1136/ bjsm.2009.058834

19. Kikukawa K, Ide J, Terakawa Y, Takada K, Morita M, Hashimoto K, Mizuta H (2016) Hypertrophic teres minor restores shoulder strength and range of external rotation in posterosuperior rotator cuff tears. J Shoulder Elbow Surg 25(11):1882-1888. https://doi. org/10.1016/j.jse.2016.04.016
20. Kim DS, Park HK, Park JH, Yoon WS (2012) Ganglion cyst of the spinoglenoid notch: comparison between SLAP repair alone and SLAP repair with cyst decompression. J Shoulder Elbow Surg 21(11):1456-1463. https://doi.org/10.1016/j.jse.2012.01.013

21. Lajtai G, Pfirrmann CW, Aitzetmüller G, Pirkl C, Gerber C, Jost B (2009) The shoulders of professional beach volleyball players: high prevalence of infraspinatus muscle atrophy. Am J Sports Med 37(7):1375-1383. https://doi.org/10.1177/0363546512455395

22. Lajtai G, Wieser K, Ofner M, Raimann G, Aitzetmüller G, Jost B (2012) Electromyography and nerve conduction velocity for the evaluation of the infraspinatus muscle and the suprascapular nerve in professional beach volleyball players. Am J Sports Med 40(10):2303-2308. https://doi.org/10.1177/0363546512455395

23. Liveson JA, Bronson MJ, Pollack MA (1991) Suprascapular nerve lesions at the spinoglenoid notch: report of three cases and review of the literature. J Neurol Neurosurg Psychiatry 54(3):241-243

24. Mall NA, Hammond JE, Lenart BA, Enriquez DJ, Twigg SL, Nicholson GP (2013) Suprascapular nerve entrapment isolated to the spinoglenoid notch: surgical technique and results of open decompression. J Shoulder Elbow Surg 22(11):e1-8. https://doi. org/10.1177/0363546512455395

25. Mallon WJ, Wilson RJ, Basamania CJ (2006) The association of suprascapular neuropathy with massive rotator cuff tears: a preliminary report. J Shoulder Elbow Surg 15(4):395-398. https ://doi.org/10.1016/j.jse.2005.10.019

26. Massimini DF, Singh A, Wells JH, Li G, Warner JJ (2013) Suprascapular nerve anatomy during shoulder motion: a cadaveric proof of concept study with implications for neurogenic shoulder pain. J Shoulder Elbow Surg 22(4):463-470. https://doi.org/10.1016/j. jse.2012.04.018

27. Melis B, DeFranco MJ, Chuinard C, Walch G (2010) Natural history of fatty infiltration and atrophy of the supraspinatus muscle in rotator cuff tears. Clin Orthop Relat Res 468(6):1498-1505. https ://doi.org/10.1007/s11999-009-1207-x

28. Montagna P, Colonna S (1993) Suprascapular neuropathy restricted to the infraspinatus muscle in volleyball players. Acta Neurol Scand 87(3):248-250

29. Paraskevas G, Tzaveas A, Papaziogas B, Kitsoulis P, Natsis K, Spanidou S (2008) Morphological parameters of the acromion. Folia Morphologica 67(4):255-260

30. Park YB, Ryu HY, Hong JH, Ko YH, Yoo JC (2016) Reversibility of supraspinatus muscle atrophy in tendon-bone healing after arthroscopic rotator cuff repair. Am J Sports Med 44(4):981-988. https://doi.org/10.1177/0363546515625211

31. Polguj M, Jędrzejewski K, Podgórski M, Topol M (2011) Morphometric study of the suprascapular notch: proposal of classification. Surg Radiol Anat 33(9):781-787. https://doi.org/10.1007/s0027 6-011-0821-y

32. Polguj M, Jędrzejewski KS, Topol M (2013) Sexual dimorphism of the suprascapular notch-morphometric study. Arch Med Sci 9(1):177-183. https://doi.org/10.5114/aoms.2013.33173

33. Polguj M, Sibiński M, Grzegorzewski A, Grzelak P, Majos A, Topol M (2013) Variation in morphology of suprascapular notch as a factor of suprascapular nerve entrapment. Int Orthop 37:2185-2192. https://doi.org/10.1007/s00264-013-2005-3

34. Reeser JC, Fleisig GS, Cools AM, Yount D, Magnes SA (2013) Biomechanical insights into the aetiology of infraspinatus syndrome. Br J Sports Med 47(4):239-244. https://doi.org/10.1136/ bjsports-2011-090918

35. Rempel DM, Diao E (2004) Entrapment neuropathies: pathophysiology and pathogenesis. J Electromyogr Kinesiol 14(1):71-75. https://doi.org/10.1016/j.jelekin.2003.09.009

36. Ringel SP, Treihaft M, Carry M, Fisher R, Jacobs P (1990) Suprascapular neuropathy in pitchers. Am J sports Med 18(1):80-86 
37. Rokito AS, Jobe FW, Pink MM, Perry J, Brault J (1998) Electromyographic analysis of shoulder function during the volleyball serve and spike. J Shoulder Elbow Surg 7:256-263

38. Ryu RK, McCormick J, Jobe FW, Moynes DR, Antonelli DJ (1988) An electromyographic analysis of shoulder function in tennis players. Am J Sports Med 16:481-485

39. Sandow MJ, Ilic J (1998) Suprascapular nerve rotator cuff compression syndrome in volleyball players. J Shoulder Elbow Surg 7(5):516-521

40. Shi LL, Freehill MT, Yannopoulos P, Warner JJ (2012) Suprascapular nerve: is it important in cuff pathology? Adv Orthop 2012:516985. https://doi.org/10.1155/2012/516985

41. Shi L, Griffith JF, Huang J, Wang D (2013) Excellent side-to-side symmetry in glenoid size and shape. Skeletal Radiol 42:17111715. https://doi.org/10.1007/s00256-013-1728-y

42. Smith AN (1995) Suprascapular Neuropathy in a Collegiate Pitcher. ATC J Athl Train 30(1):43-46

43. Tapadia M, Mozaffar T, Gupta R (2010) Compressive neuropathies of the upper extremity: update on pathophysiology, classification, and electrodiagnostic findings. J Hand Surg Am 35(4):668-677. https://doi.org/10.1016/j.jhsa.2010.01.007

44. Teunis T, Lubberts B, Reilly BT, Ring D (2014) A systematic review and pooled analysis of the prevalence of rotator cuff disease with increasing age. J Shoulder Elbow Surg 23(12):19131921. https://doi.org/10.1016/j.jse.2014.08.001
45. Ticker JB, Djurasovic M, Strauch RJ, April EW, Pollock RG, Flatow EL, Bigliani LU (1998) The incidence of ganglion cysts and other variations in anatomy along the course of the suprascapular nerve. J Shoulder Elbow Surg 7(5):472-478. https://doi. org/10.1016/S1058-2746(98)90197-5. (https://doi.org/10.1007/ s11999-012-2494-1)

46. Wang DH, Koehler SM (1996) Isolated infraspinatus atrophy in a collegiate volleyball player. Clin J Sport Med 6(4):255-258

47. Witvrouw E, Cools A, Lysens R, Cambier D, Vanderstraeten G, Victor J, Sneyers C, Walravens M (2000) Suprascapular neuropathy in volleyball players. Br J Sports Med 34:174-180. https://doi. org/10.1136/bjsm.34.3.174

48. Won HJ, Won HS, Oh CS, Han SH, Chung IH, Yoon YC (2014) Morphological study of the inferior transverse scapular ligament. Clin Anat 27(5):707-711. https://doi.org/10.1002/ca.22283

49. Yao L, Mehta U (2003) Infraspinatus muscle atrophy. implications? Radiology 226(1):161-164. https://doi.org/10.1148/radio 1.2261011818

50. Young SW, Dakic J, Stroia K, Nguyen ML, Harris AL, Safran MR (2015) High incidence of infraspinatus muscle atrophy in elite professional female tennis players. Am J Sports Med 43(8):1989_ 1993. https://doi.org/10.1177/0363546515588177 